\title{
MODERNIZACIÓN TECNOLÓGICA Y CAMBIOS ORGANIZACIONALES EN LA INDUSTRIA MAQUILADORA
}

\author{
Por \\ Jorge Carrillo Viveros \\ Miguel Ángel Ramírez*
}

\section{RESUMEN}

Actualmente, la importancia que representa para México la industria maquiladora de exportación (MB) puede resumirse en los siguientes cuatro puntos: primero, es la segunda fuente de divisas para el país (después del petróleo); la tasa de absorción de fuerza de trabajo excede a la de los demás sectores de la economía; su crecimiento en el número de establecimientos; la complejidad y modernización que muestra en los últimos años. Bajo este panorama, se justifica la atención que se le presta a la IME ante el probable Tratado Trilateral de Acuerdo de Libre Comercio, con el que se espera continuará su expansión, por lo que se hace necesario realizar estudios que fortalezcan el aprovechamiento de oportunidades que pudiera ofrecer la IME.

Es en este sentido que en este trabajo se analizan algunos aspectos relacionados con la tecnología en plantas maquiladoras, especialmente aquellas con nuevas formas de organización en el trabajo. Las hipótesis que se manejan son dos: existe una amplia y heterogénea difusión de la tecnología en la IME; existen diferencias significativas en la estructura organizativa entre plantas que utilizan nuevas tecnologías blandas o métodos de organización flexible del trabajo, y plantas de tipo más tradicional. Para la realización de este trabajo se llevó a cabo una encuesta probabilística en Ciudad Juárez, Tijuana y Monterrey.

Algunas de las conclusiones a las que se llegan reflejan que la IME no sólo es importante por las divisas y empleos que genera a nivel nacional, sino también por los impactos que propicia a nivel regional. Las características actuales de la ME la definen por su dinamismo, heterogeneidad y reestructuración. Dentro de la reestructuración se observa: la difusión de procesos automatizados; los cambios en el empleo, y la proliferación de la flexibilización organizacional.

En lo que se refiere a la flexibilización organizacional en la IME, los resultados de este trabajo permiten señalar que existe un proceso generalizado, pero que éste no es homogéneo entre las distintas actividades económicas.

\begin{abstract}
Presentely the magnitude of the maquiladora industry in Mexico can be summarized: It is the first source of foreign exchange (after oil); the employment absorption rate is larger than in the other economic activities of the country; and it shows longer increase in the number of the plants. With this background, the attention of the maquila industry into the probably Free Trade
\end{abstract}

* Investigadores de El Colegio de la Frontera Norte. 
Agreementis justified, because the expectation is that the maquila will continue to expand, and is therefore necessary to do research in order to take advantage of opportunities offered by the maquila.

This paper analizes some aspects of technology in maquila plants, specially regarding new work organization. The hypotheses are two: there exists an vast, heterogenity diffusion of technology throughout the maquila plants, alternatively, there are significant differences in organizative structure between plants wich use new technologies of flexible work organization models and plants that are more traditional. To carry out this research a probabilist inquiry was conducted in Ciudad Juarez, Tijuana and Monterrey.

Conclusions show that the maquiladora industry in very important as a source of foreign exchange and employment at the nacional level, but also is important because of the impact at a regional level. At present the characteristics of the maquiladora industry are defined by dynamism, heterogeneity and restructuring, and this includes the diffussion of automated processes, changes in employment and the growth of the organizational flexibility.

\section{INTRODUCCIÓN ${ }^{1}$}

La industria maquiladora de exportación (en adelante IME) es una de las industrias más dinámicas en su crecimiento dentro de México. La importancia de la IME puede resumirse en los siguientes puntos: 1) su constitución actual como la segunda fuente de divisas para el país después del petróleo (1,500 millones de dólares en 1985, lo que equivalió al $5 \%$ de los ingresos totales en cuenta corriente); 2) una tasa de absorción de fuerza de trabajo que excede multiplicativamente al empleo que generan los demás sectores de actividad económica (18.5\% anual de 1984 a 1988), y que representa actualmente al $18 \%$ de la población económicamente activa en la industria de la manufactura; 3 ) el crecimiento en el número de establecimientos, y 4) su complejidad y modernización.

La enorme atención que ha cobrado la IME, tanto en México como en Estados Unidos y recientemente en Canadá, se debe al sostenido dinamismo de su crecimiento y a la envergadura de su tamaño. Para agosto de 1990, según reporta INEGI, existían 1909 establecimientos que ocupaban a 459,837 personas y generaron, entre enero y agosto de ese año $\$ 2,200$ millones de dólares.

La justificada atención que se le ha dado a la IME a partir del probable Tratado Trilateral de Libre Comercio (TTLC) se debe al comportamiento en el crecimiento que ha tenido dicha industria, a las proyecciones que demuestran que continuará dicha expansión, y a la importancia económica

1 Los datos empíricos de este trabajo forman parte del proyecto de investigación: "Mercados de trabajo en las actividades maquiladoras", auspiciado por la Secretaría del Trabajo y Previsión Social. Todas las ideas expresadas en el mismo son responsabilidad exclusiva del autor. La estadística estuvo a cargo de Jorge Santibañez y el procesamiento de datos a cargo de Francisco Barraza. 
que guarda la IME en el contexto nacional. Los escenarios futuros mencionan que es posible que se acelere el proceso de maquilización de la industria en México a partir del TTLC. Lo anterior coadyuva a una necesaria comprensión del desarrollo tecnológico en la IME y a fortalecer los estudios que apunten sobre el aprovechamiento de oportunidades.

En este sentido, en este trabajo se analizan algunos aspectos relacionados con la tecnología en plantas maquiladoras, especialmente con nuevas formas de organización del trabajo.

Este documento está estructurado de la siguiente manera: 1) presentación sucinta de la metodología, 2) breve exposición de rasgos sobresalientes de la IME, 3) resultados de la investigación sobre tecnología,4) contraste de variables en plantas con mayor y menor flexibilidad organizativa y 5) conclusiones generales.

\section{METODOLOGÍA}

En este trabajo se analiza la IME en México a través de una encuesta probabilista que realizó El Colegio de la Frontera Norte en febrero de 1990 en diversas plantas maquiladoras establecidas en Tijuana, Ciudad Juárez y Monterrey, pertenecientes a las actividades de la electrónica, las autopartes y el vestido.

Se seleccionaron aleatoriamente 210 plantas maquiladoras las cuales representaron a 358 plantas. Las hipótesis de trabajo que se manejan en este documento son dos: 1) existe una amplia y heterogénea difusión de la tecnología en la IME, y 2) hay diferencias significativas en la estructura organizativa entre plantas que utilizan nuevas tecnologías blandas o métodos de organización flexible del trabajo, y plantas de tipo más tradicional.

La plausibilidad de estas ideas radica en que estudios previos de caso sobre la IME reportan un importante proceso de difusión de nuevas tecnologías, con lo cual se supone que se presentan una serie de efectos en el empleo, en las características de los trabajadores y en las estructuras organizativas de las plantas según el nivel de tecnología implementado.

Para revisar la primera hipótesis sobre la difusión de la tecnología, se analizan variables como porcentaje de rechazos a la producción, participación en el justo a tiempo y número de unidades programables a partir de los promedios del total de las plantas analizadas. Y, para examinar las diferencias entre plantas con mayor y menor flexibilidad, se crean dos. grupos a partir de la participación de la mano de obra directa en ocho actividades relacionadas con la forma de organización de trabajo. Se consideraron como plantas con "mayor flexibilidad" (MAYORFLEX) aquellas cuyo personal directo se encuentra involucrado en una proporción 
superior al 30\%, como promedio de las ocho actividades relacionadas con la organización flexible; ${ }^{2}$ y como plantas con "menor flexibilidad" (MENORFLEX) aquellas con $30 \%$ o menos de su personal directo involucrado en esas mismas ocho actividades.

Bajo este criterio se encontró que $27 \%$ de las plantas fueron clasificadas como de "mayor flexibilidad" y 73\% como de "menor flexibilidad". El promedio de participación de los trabajadores en varias de las actividades resultó alto y la mayor participación se presenta en el trabajo en grupo, seguida del involucramiento en el trabajo, los cuales alcanzaron a más del $50 \%$ del total de la mano de obra directa involucrada en esas actividades en las 358 plantas.

Una vez formados los grupos se analizan los siguientes aspectos: datos generales de las plantas (empleo, antigüedad y costo de la mano de obra), características de la fuerza de trabajo (origen, experiencia laboral, antigüedad, edad, sexo y escolaridad) y aspectos de la estructura organizativa (niveles jerárquicos, personal directo, calificación y salarios).

\section{ACERCA DE LA INDUSTRIA MAQUILADORA DE EXPORTACIÓN}

La IME surgió en México en 1965 con el establecimiento del programa gubernamental denominado de Industrialización Fronteriza. Mediante este programa se permitía tanto la importación de insumos y componentes a nuestro país, como la exportación de los productos libres de impuestos, excepto del valor agregado. Esta reducción de aranceles ha permitido el traslado de importantes segmentos productivos de Estados Unidos hacia México, fundamentalmente en la búsqueda del abaratamiento de los costos de producción.

El desarrollo de la IME ha sido complejo en la presente década. Transformaciones sectoriales, productivas y organizacionales, entre otras, han llevado a considerar que dicha industria se encuentra en una nueva etapa. Esto ha permitido, en la actualidad, presentar a la maquiladora en forma segmentada: por un lado, sectores y empresas de punta con procesos productivos modernizados a través de la incorporación de innovaciones tecnológicas duras y blandas; y por otrolado, sectores y empresas atrasadas en donde prevalecen los procesos tradicionales de ensamble.

2 Las ocho actividades son: 1) mantenimiento del equipo y maquinaria, 2) diagnóstico y solución de problemas de producción, 3) control o inspección de calidad, 4) círculos, grupos o juntas de calidad, 5) equipos de trabajo, grupos de trabajo o producción en grupos; 6) multicalificación, 7) rotación de tareas y operaciones, 8 ) involucramiento en el trabajo. 
Existe una literatura especializada que evidencia que existen dos fases en la historia de la industria maquiladora. Los trabajos de Carrillo (1986), González-Aréchiga y Barajas (1987), Wilson (1989), González-Aréchiga y Ramírez (1989 y 1990) y Brown y Domínguez (1989) son una buena muestra de ello. Revisando algunos de los rasgos más generales de estas etapas tenemos los siguientes:

La primera etapa abarca la década de 1960 a 1970, y está caracterizada por el inicio del Programa de Industrialización Fronteriza, su ampliación hacia el interior del país, y la consolidación a través de su crecimiento. Las tasas de crecimiento en esta fase tuvieron fuertes altibajos, sin embargo, de 1974 a 1980 el valor agregado de la IME creció a una tasa anual del $15.83 \%$, el empleo a una del $7.84 \%$ y el número de establecimientos a una del $5.29 \%$. En términos productivos, la industria se caracterizó por ser de ensamble intensivo en mano de obra, con bajo uso de tecnología, con actividades parciales y repetitivas y con una organización simple del trabajo. Acompañando a esta forma de proceso de producción se presentaba una oferta abundante de empleo, un empleo femenino y una baja exigencia de capacitación dentro del trabajo. Los bajos índices de rotación, las exigencias mayores en la selección del personal y el bajo nivel de relación entre cl sector productivo y educativo fucron característicos de esta primera etapa industrial de la IME. La literatura menciona el establecimiento de una industria con baja tecnología y trabajo no calificado.

La segunda etapa abarca desde la década de los ochenta hasta el presente, $\mathrm{y}$ se ha caracterizado por su sistemática transformación en diferentes órdenes, entre los más conspicuos se encuentran: la mayor composición técnica del capital, el desarrollo de procesos automatizados, el peso y participación de los sectores productivos de la electrónica y de las autopartes, el incremento del sector masculino en el trabajo, la flexibilidad en la organización de la producción y del trabajo y la regulación del empleo. Las tasas de crecimiento en esta fase fueron más altas que en la etapa anterior. Las tasas anuales de crecimiento, entre 1981 y 1989, fueron del orden del $16.96 \%$ en el valor agregado; de $15.60 \%$ en el empleo y de $14.18 \%$ en el número de establecimientos. Es decir, 1.6 veces mayores en los establecimientos que en la década anterior, 0.9 mayor en los trabajadores y 0.07 en el valor agregado, respectivamente.

En términos productivos, las empresas se pueden calificar en tres tipos según Patricia Wilson: de ensamble tradicional, "postfordistas" y manufactureras (Wilson, 1989). Cada uno de cstos tipos está caracterizado por una diferente intensidad en el empleo de mano de obra. En términos generales, los tres tipos de empresas presentan, aunque claramente en forma heterogenca, el uso de nuevas tecnologías y nuevas formas de 
organización del trabajo y de la producción. El trabajo, también en términos muy generales, se recalifica por el grado de responsabilidad y participación que exige a los trabajadores un mayor involucramiento en la producción.

Al parecer, en esta segunda etapa se contrae la oferta en el empleo y se equilibra el uso de mujeres y hombres en la producción; se empieza a desarrollar un mayor esfuerzo en el área de la capacitación; se incrementan sustancialmente los índices de rotación, al grado de convertirse en uno de los problemas fundamentales en dicha industria; bajan las exigencias en la selección de personal pero al mismo tiempo se incrementa la relación entre el sector productivo y educativo, particularmente en los niveles técnico y profesional. Se considera que en esta etapa el trabajo se ha recalificado y que han aparecido nuevas habilidades.

El carácter segmentado e internacionalizado del proceso de producción ha provocado que la IME se haya caracterizado por el uso intensivo de la mano de obra, motivo por el cual las maquiladoras han sido concebidas como centros de costos y no de utilidades. La singularidad de ser fundamentalmente plantas orientadas hacia la exportación y con uso intensivo de la mano de obra, así como el tratamiento arancelario específico que le otorga el gobierno, ha llevado a considerarlas no sólo como una industria homogénea sino como industria en sí misma. Si bien esto es cierto, la IME lejos de ser una industria, propiamente dicho, es un conjunto de actividades económicas que se integran verticalmente a diversas industrias (electrónica, automotriz, mueblera, de la confección, etc.). Este simple hecho deviene en una singular heterogeneidad de los procesos de producción y de organización. Asimismo, las oportunidades de reducir costos relativos de producción ha provocado el fenómeno de las relocalizaciones productivas, las cuales rebasan la simple característica de "mano de obra barata sin calificación". Este hecho lleva a considerar que el término "maquiladora", más que referirse a un concepto productivo, debe relacionarse con uno arancelario. Esto se ejemplifica con el hecho de que una planta "maquiladora" puede dedicarse, total o parcialmente, al ensamble o a la manufactura.

\section{ACERCA DE LAS TECNOLOGÍAS BLANDAS: NUEVAS FORMAS DE GESTIÓN DE LA MANO DE OBRA}

Revisemos brevemente tres estudios que arrojan resultados empíricos sobre la organización flexible en el trabajo.

Palomares y Mertens (1985) encuentran en las industrias electrónicas entrevistadas la utilización de las técnicas cero error y justo a tiempo; un 
incremento del personal calificado y cambio en las propias actividades del personal (incluyendo incrementos en la capacitación); técnicas de involucramiento en el empleo; incremento de los salarios (a través de bonos de puntualidad, asistencia, etc.) "a cambio" de responsabilidad-compromiso y disminución de la rotación, y una cierta reducción en el empleo.

Wilson (1989) reporta una utilización del justo a tiempo del orden de $42 \%$ ( $n=12$ plantas) en plantas "postfordistas", del $9 \%$ en manufactureras "fordistas"...(n=32) y del $25 \%$ en ensambladoras intensivas en trabajo $(n=24)$. Los resultados de prácticas de multicalificación (o polivalencia en el trabajo) fueron del 50,39 y $21 \%$, respectivamente. De participación de los trabajadores: 75,29 y $46 \%$; y de continuidad en el control de la calidad: 92,81 y $88 \%$, respectivamente.

Carrillo (1990) encuentra una amplia difusión de técnicas de organización flexible. En las once plantas maquiladoras de autopartes visitadas en Ciudad Juárez y Nuevo Laredo (al igual que en seis plantas no maquiladoras del interior del país) existen por lo menos dos técnicas organizacionales adaptadas. En cinco plantas se ha implementado el justo a tiempo, en nueve el control estadístico de proceso, en diez los círculos (o juntas) de calidad, en seis los grupos o equipos de trabajo, y en dos el involucramiento en el empleo. Se reportan también la existencia de nuevas habilidades y la ampliación de los programas de capacitación de la mano de obra, así como un amplio uso de hombres en procesos simples de ensamble y en procesos de manufacturas (dentro de las maquiladoras).

Las conclusiones generales que se pueden establecer de estos tres trabajos son que existe un proceso de incorporación de nuevas tecnologías duras y blandas en plantas maquiladoras, tanto en la frontera como en el interior del país. Que si bien hay una amplia difusión de nuevas tecnologías, coexisten procesos tradicionales de ensamble con procesos de tecnologías avanzadas.

La evidencia empírica presentada en estos trabajos constata que sí es compatible el uso de nuevas tecnologías en la industria maquiladora; que contrario a la tesis de la relocalización de plantas hacia los países de origen por la adaptación de procesos de producción flexible, México representa una opción única dentro del proceso de automatización y flexibilización ya que la combinación de diversos factores de localización permite responder a las distintas oportunidades de mercado. En este sentido cobra relevancia el hallazgo de la proliferación rápida y novedosa de procesos de manufactura en la maquiladora, sef́alada en varios trabajos, pero analizada con mayor sistematicidad por Wilson (1989).

A través de métodos directos o indirectos todos estos estudios reportan un aumento de la eficiencia en las plantas, producto del mejoramiento en la calidad y productividad del trabajo. 
A pesar de los signos positivos que acompanan este proceso de modernización industrial, y de que cada vez se vuelve más complejo, existen evidencias que permiten señalar la necesidad de una mejor evaluación del parámetro tecnológiço. Dentro de este parámetro una de las áreas menos atendidas en la investigación sobre la IME es la flexibilidad organizacional. A continuación se revisan algunos aspectos relacionados con ella.

Las exportaciones de maquiladoras están sujetas a una gran competencia intemacional. Algunos de los indicadores centrales que en la actualidad son utilizados para conocer el grado de competitividad de las plantas son el porcentaje de rechazos en la producción y el justo a tiempo. ${ }^{3}$ Estas técnicas generalmente van unidas a otras más relacionadas con la modernización en la gestión de la mano de obra.

a) rechazos en la producción. Se puede mencionar que éstos son uno de los mejores indicadores del control de calidad, y que es un sistema ampliamente utilizado en las industrias que concurren en el mercado internacional altamente competitivo, como es el caso del sector automotriz. En la encuesta de el COLEF resultó que la mayoría de las plantas - más del $60 \%$ de ellas-, se encontraban en el rango 0-5\% de rechazos. Llamó laatención el caso de las autopartes que tuvo el índice más bajo de rechazos a la producción.

b) Justo a tiempo. Se encontró una amplia difusión de este sistema. A partir de la encuesta se lograron distinguir dos segmentos tecnológicos derivados de la implementación del sistema justo a tiempo en las maquiladoras. La proliferación de dicho sistema se presentó con mayor fuerza en las ciudades fronterizas, donde más de la mitad del total de las plantas trabajaban con dicha técnica en más del $75 \%$ de sus productos. Las plantas que laboran con un rango bajo de días de inventario (esto es, entre menos de 1 y hasta 9 días - contando desde que entran los componentes hasta que salen los productos de la planta-), fueron la mayoría de las tres actividades económicas analizadas.

c) Unidades programables. Para medir la tecnología dura se preguntó el número de unidades programables con que contaba la planta en cada departamento. El promedio de unidades programables por planta resultó diferente entre los sectores productivos: 22 unidades en la electrónica, 12 en las autopartes y 3.4 en el vestido. Se encontró una alta heterogeneidad interna en cada sector productivo. En cuanto a su distribución según tipo de unidad se cuenta con la siguiente información: de 6,605 unidades programables en las 358 plantas analizadas, el $79.6 \%$ de las unidades eran

3 De su producto principal durante la semana anterior a la entrevista. 
máquinas herramientas de control numérico, $19.7 \%$ robots, $0.4 \%$ equipo programable para traslado y $0.27 \%$ otro tipo de unidad.

La industria de la electrónica se encuentra a la vanguardia en el número de unidades programables que incorpora (computadoras, máquinas-herramienta de control numérico, robots y equipo de traslado). En el área de inserción, se encontró en las plantas de ensamble de televisores se utilizan una gran cantidad de máquinas de inserción automática que, dado el nivel de operaciones que realizan y la flexibilidad de las mismas, algunos expertos las consideran como robots (sin brazos).

Acerca del número de empleados por unidad programable resultó que, como era de esperarse, hay más empleados por máquinas en el sector de la electrónica (64.5\%) que en las autopartes (51.1\%) o que en el vestido (31.7\%). También aquí existe heterogeneidad interna. El mayor número de empleados por unidades programables en las industrias de vanguardia tecnológica, como la electrónica, se debe a que son sensiblemente de mayor tamaño que las del vestido. Así, mientras que es aproximadamente dos veces mayor el número de unidades por trabajador en el vestido que en la electrónica, es seis veces mayor el tamaño de empleo promedio en este último sector que en el primero.

d) Organización flexible. Se define por organización flexible la presencia de ocho elementos organizativos dentro de las empresas. ${ }^{4}$ Se parte del supuesto de que a mayor porporción de la mano de obra participando en cada uno de esos ocho elementos, es mayor la flexibilidad.

Anteriormente se pensaba que dichas técnicas sólo existían en algunas plantas de vanguardia tecnológica y que a mayor tecnología mayor flexibilidad en la organización del trabajo. Los datos de la encuesta mostraron que las ideas anteriores no son correctas. Los datos confirmaron una clara tendencia, en muchas de las plantas, hacia el involucramiento de la mano de obra independientemente del nivel de tecnología dura. Se encontró que la participación de la mano de obra en técnicas flexibles no estuvo asociada ni con la antigüedad de las plantas ni con la automatización en los equipos. En general, mientras que las entrevistas a profundidad reportaron un proceso a largo plazo que busca incorporar al $100 \%$ de los empleados en la mayoría de las nuevas formas de organización flexible, la información sobre implementación de equipo automatizado indica que su uso es muy heterogéneo entre las plantas.

Las actividades organizativas más difundidas en las plantas maquiladoras fueron el trabajo en equipo y las técnicas de involucramiento en el

\footnotetext{
4 Consúltese la nota de pie número 2.
} 
empleo: el $56.8 \%$ de la mano de obra directa, en el total de plantas, participaba en la primera actividad y el $53.2 \%$ en la segunda. Un segundo grupo estuvo conformado por las técnicas que tuvieron más del $30 \%$ de su mano de obra directa participando en: rotación de tareas, círculos de calidad y multicalificación.

Acerca de la participación del personal directo en la organización flexible, según las actividades económicas, se presentaron diferencias interesantes. Fueron mayores los promedios de participación en el mantenimiento de equipo y maquinaria en las autopartes (18.9\%) y en el vestido $(12.5 \%)$ que en la electrónica. En el diagnóstico y solución de problemas participaron el $21.4 \%$ en la electrónica y el $26.5 \%$ en las autopartes. En este último sector es donde se le otorga mayor importancia a la calidad: $27.2 \%$ en control de calidad y $49.9 \%$ en los grupos de calidad. En segundo lugar es en la electrónica y por último en el vestido (véase cuadro 1).

En el trabajo en grupo, nuevamente, es en las autopartes donde es más elevado el porcentaje de participación de la mano de obra directa (74.6\%). Sobresale también la electrónica con 51.6\%. Salvo las prácticas de multicalificación, en donde fue mayor la participación en la electrónica (38.8 vs. 28.5 en las autopartes y 26.7 en el vestido), las autopartes superan a los otros dos sectores en la rotación de tareas y en el involucramiento en el trabajo.

Por lo tanto, todo parece indicar que es en las autopartes donde se presenta, en promedio, la mayor participación del personal directo en forma de organización flexible en el trabajo. En general, los altos porcentajes sugieren una difundida flexibilización en el proceso de organización.

De los datos anteriores se deriva que existe una reestructuración organizacional generalizada que apunta hacia un mayor involucramiento de los trabajadores en actividades que anteriormente pertenecían a distintos departamentos, muchos de ellos para personal indirecto. La transformación organizativa, a pesar de su amplia difusión, mantiene diferencias internas considerables, como es el caso de algunas de las variables expuestas con anterioridad, lo que da firmeza a la hipótesis de que existen diversos modelos de maquiladora que rebasan los cortes de ciudad y sector, es decir, que para comprender los niveles y modelos organizativos y tecnológicos, no basta con entender las diferencias regionales y sectoriales, las cuales ya en sí mismas reflejan varias industrias maquiladoras y no sólo una.

\section{FLEXIBILIDAD ORGANIZACIONAL}

En esta sección se examina la hipótesis que menciona que existen diferencias significativas en la estructura organizativa entre plantas que utilizan nuevas tecnologías blandas o métodos de organización flexible del 
CUADRO 1. Porcentaje de participación del personal directo en la organización flexible según actividad económica (promedios).

\begin{tabular}{|c|c|c|c|}
\hline Prestaciones & Electrónica & Autopartes & Vestido \\
\hline $\begin{array}{l}\text { Mantenimiento de equipo } \\
\text { y maquinaria }\end{array}$ & $\begin{array}{c}7.6 \\
(9.8) \\
117\end{array}$ & $\begin{array}{c}18.9 \\
(31.6) \\
33\end{array}$ & $\begin{array}{c}12.5 \\
(23.1) \\
42\end{array}$ \\
\hline $\begin{array}{l}\text { Diagnóstico y solución } \\
\text { de problemas }\end{array}$ & $\begin{array}{c}21.4 \\
(29.2) \\
134\end{array}$ & $\begin{array}{c}26.5 \\
(36.7) \\
42\end{array}$ & $\begin{array}{c}6.4 \\
(7.9) \\
40\end{array}$ \\
\hline Control de calidad & $\begin{array}{c}18.2 \\
(26.4) \\
171\end{array}$ & $\begin{array}{c}27.2 \\
(32.8) \\
49\end{array}$ & $\begin{array}{c}7.8 \\
(10.5) \\
55\end{array}$ \\
\hline $\begin{array}{l}\text { Grupos o juntas de } \\
\text { calidad }\end{array}$ & $\begin{array}{c}33.2 \\
(35.0) \\
140\end{array}$ & $\begin{array}{c}49.9 \\
(40.6) \\
36\end{array}$ & $\begin{array}{c}19.9 \\
(31.3) \\
31\end{array}$ \\
\hline Producción en grupo & $\begin{array}{c}51.6 \\
(38.8) \\
96\end{array}$ & $\begin{array}{c}74.6 \\
(28.5) \\
29\end{array}$ & $\begin{array}{c}44.3 \\
(40.8) \\
33\end{array}$ \\
\hline $\begin{array}{l}\text { Multicalificación del } \\
\text { trabajador }\end{array}$ & $\begin{array}{c}38.8 \\
(32.4) \\
133\end{array}$ & $\begin{array}{c}28.5 \\
(31.7) \\
37\end{array}$ & $\begin{array}{c}26.7 \\
(32.3) \\
45\end{array}$ \\
\hline $\begin{array}{l}\text { Rotación de tareas y } \\
\text { operaciones }\end{array}$ & $\begin{array}{c}40.3 \\
(32.8) \\
163\end{array}$ & $\begin{array}{c}44.5 \\
(34.8) \\
39\end{array}$ & $\begin{array}{c}23.6 \\
(27.1) \\
47\end{array}$ \\
\hline $\begin{array}{l}\text { Involucramiento en el } \\
\text { trabajo }\end{array}$ & $\begin{array}{c}52.6 \\
(41.3) \\
124\end{array}$ & $\begin{array}{c}59.5 \\
(37.4) \\
34\end{array}$ & $\begin{array}{c}47.5 \\
(40.8) \\
35\end{array}$ \\
\hline \multicolumn{4}{|c|}{$\begin{array}{l}\text { FUENTE: Encuesta a plantas maquiladoras, Secretaría del Trabajo y Previsión Social } \\
\text { y El Colegio de la Frontera Norte. Proyecto: "Mercados de Trabajo en las } \\
\text { Actividades Maquiladoras", } 1990 . \\
\text { NOTA: Los números entre paréntesis son las desviaciones estándar } \\
\text { correspondientes, y el siguiente debajo es el número de casos (plantas } \\
\text { analizadas). }\end{array}$} \\
\hline
\end{tabular}


trabajo, y plantas de tipo más tradicional. Para ello se revisan diversas variables que a continuación se presentan agrupadas en características de las plantas, de la mano de obra y de la estructura organizacional.

\section{Características de las plantas}

\section{a) Tamaño del empleo}

Con respecto al tamaño de empleo de las plantas según su grado de flexibilidad se observa que las plantas con "mayor flexibilidad" (en adelante MAYORFLEX) son en su mayoría de gran tamaño: $47.7 \%$ de estas plantas tiene más de 500 empleados. Las plantas con "menor flexibilidad" (en adelante MENORFLEX), en cambio, no presentan grandes diferencias en su distribución por rangos de empleo, aunque se observa una ligera mayoría del $37.8 \%$ en el rango de 101 a 500 empleados.

Estos resultados parecen confirmar que la flexibilidad organizacional es, entre otras cosas, una estrategia para resolver los problemas derivados del "gigantismo" en el empleo.

CUADRO 2. Tamaño del empleo de las plantas según su grado de flexibilidad.

\begin{tabular}{|c|c|c|c|c|}
\hline \multirow{2}{*}{$\begin{array}{l}\text { Empleo } \\
\text { Rangos de empleo }\end{array}$} & \multicolumn{2}{|c|}{$\begin{array}{l}\text { Plantas con menor } \\
\text { flexibilidad }\end{array}$} & \multicolumn{2}{|c|}{$\begin{array}{l}\text { Plantas con mayor } \\
\text { flexibilidad }\end{array}$} \\
\hline & No. & $\%$ & No. & $\%$ \\
\hline $1-100$ & 64 & 30.8 & 10 & 12.9 \\
\hline $101-500$ & 79 & 37.8 & 31 & 39.4 \\
\hline Más de 500 & 66 & 31.5 & 37 & 47.7 \\
\hline Total & 209 & 100.0 & 78 & 100.0 \\
\hline
\end{tabular}

FUENTE: Misma del cuadro 1.

\section{b) Antigüedad}

En relación con la antigüedad de las plantas según su flexibilidad, las plantas con MAYORFLEX se establecieron en su mayoría antes de 1984. Lo que parece indicar una mayor tendencia entre las plantas antiguas a reestructurarse mediante la incorporación de métodos de organización 
flexible más que de nuevas tecnologías (ya que fueron comparados los datos entre ambos segmentos). Por lo demás, se confirma que la reestructuración -en el sentido amplio de tecnología blanda - se presenta tanto en plantas ya establecidas como en plantas que inician operaciones.

CUADRO 3. Antigüedad de las plantas según su grado de flexibilidad.

\begin{tabular}{|c|c|c|c|c|}
\hline \multirow[b]{2}{*}{ Rangos de antigüedad } & \multicolumn{2}{|c|}{$\begin{array}{l}\text { Plantas con menor } \\
\text { flexibilidad }\end{array}$} & \multicolumn{2}{|c|}{$\begin{array}{l}\text { Plantas con mayor } \\
\text { flexibilidad }\end{array}$} \\
\hline & No. & $\%$ & No. & $\%$ \\
\hline $1966-1978$ & 69 & 32.5 & 18 & 22.9 \\
\hline $1979-1983$ & 45 & 21.4 & 26 & 33.7 \\
\hline 1984-1989 & 98 & 46.2 & 34 & 43.4 \\
\hline Total & 211 & 100.0 & 78 & 100.0 \\
\hline
\end{tabular}

FUENTE: Misma del cuadro 1.

\section{Características de la mano de obra ${ }^{5}$}

\section{a) Origen}

La proporción de trabajadores de origen rural comparada con los de origen urbano resultó no significativa. Ya que las plantas con MAYORFLEX tuvieron un porcentaje de mano de obra de origen rural del $45.3 \%$ y las de MENORFLEX del $42.4 \%$. Esta diferencia coincide con la encontrada entre plantas clasificadas según su grado de tecnología dura (ausentes en este documento), lo que confirma que no hay diferencias significativas entre uso de tecnología y origen de la mano de obra utilizada.

\section{b) Experiencia laboral}

Con referencia a la experiencia laboral se observan diferencias. En promedio las plantas con MENORFLEX ocupan un porcentaje de trabajadores con experiencia laboral anterior ligeramente superior $(62.9 \%)$ al que reportan las plantas con MAYORFLEX (59.3\%). Este resultado también coincide con lo reportado para las plantas clasificadas según su tecnología.

5 Esta sección forma parte de un trabajo inédito más amplio en coautoría con Miguel Ángel Ramírez: "Organización y tecnología en las actividades maquiladoras". Reporte de investigación. El Colegio de la Frontera Norte, diciembre de 1990. 
Lo que se puede resumir señalando que, probablemente, la implementación de flexibilidad organizacional no está asociada con la experiencia laboral anterior, lo cual cuestiona la idea generalizada de que la mano de obra sin experiencia laboral es más proclive a la flexibilidad organizacional.

\section{c) Antigüedad en el empleo}

El promedio de antigeüdad laboral es ligeramente más alto en las plantas con MENORFLEX ( 2.3 años) que en las plantas de MAYORFLEX ( $1.9 \%$ años). Esto parece cuestionar otra idea generalizada de que las plantas con métodos de organización flexible producen un ambiente laboral más armónico que permite un arraigo mayor de su fuerza de trabajo.

\section{b) Edad de los trabajadores}

La edad promedio del personal ocupado no presentó grandes diferencias. En las plantas con MENORFLEX este promedio fue de 23.4 años, mientras que en las de MAYORFLEX fue de 22.4 afios. Este resultado es similar al encontrado en el caso de las plantas clasificadas según su grado de tecnología. Esto confirma, por un lado, la joven edad de los trabajadores directos de las maquiladoras y, por el otro, que no es una variable asociada con la flexibilidad en el trabajo.

\section{e) Sexo}

Por lo que se refiere al género del personal ocupado, los datos para las plantas según flexibilidad son consistentes con los encontrados en las plantas según su tecnología, en el sentido de que el personal directo en las maquiladoras continúa siendo en su mayoría mujeres. Pero también se observan diferencias en la proporción de hombres ocupados en uno y otro tipo de sectores según flexibilidad. El porcentaje de hombres es más alto en las plantas con MAYORFLEX (47.8\%) que en las plantas con MENORFLEX (36.5\%). Lo que puede estar indicando que la introducción de métodos de organización flexible, al igual que los procesos automatizados, es otra de las causas del cambio en la composición de los sexos en las maquiladoras.

\section{f) Escolaridad}

La escolaridad es otra de las características del personal directo que no reporta diferencias significativas. Mientras que en las plantas de MENORFLEX el promedio de educación formal es de 6.5 años, en las plantas de MAYORFLEX 
CUADRO 4. Características de la mano de obra en las plantas según su flexibilidad.

\begin{tabular}{lcc}
\hline & $\begin{array}{c}\text { Plantas con menor } \\
\text { flexibilidad }\end{array}$ & $\begin{array}{c}\text { Plantas con mayor } \\
\text { flexibilidad }\end{array}$ \\
\hline Origen urbano & & \\
Promedio (\%) & 42.4 & 45.3 \\
Desviación estándar & 32.3 & 29.6 \\
Número de plantas & 196 & 74 \\
Experiencia laboral anterior & & \\
Promedio (\%) & 62.9 & 59.3 \\
Desviación estándar & 29.8 & 24.9 \\
Número de plantas & 183 & 72 \\
Antigüedad & & \\
promedio & 2.3 & 1.9 \\
Desviación estándar & 2.7 & 2.4 \\
Número de plantas & 211 & 77 \\
Edad & & \\
Promedio (años) & 23.4 & 22.4 \\
Desviación estándar & 5.4 & 4.1 \\
Número de plantas & 209 & 77 \\
Hombres & & \\
Promedio (\%) & 36.5 & 47.8 \\
Desviación estándar & 29.7 & 25.0 \\
Número de plantas & 200 & 75 \\
Escolaridad & & 6.6 \\
Promedio (años) & 6.5 & 77 \\
Desviación estándar & 1.6 & \\
Número de plantas & 211 & \\
\hline
\end{tabular}

FUENTE: Misma del cuadro 1.

ese promedio resultó de 6.6 años. Este resultado también es similar al que se reporta para las plantas con menor y mayor tecnología.

\section{g) Costo de la mano de obra}

En este aspecto se observa una notable diferencia entre las plantas según su flexibilidad. En las plantas con MAYORFLEX la mayoría de ellas o el $57 \%$, tuvieron un costo de mano de obra menor al $20 \%$ de sus costos totales de producción; mientras que las plantas con MENORFLEX sólo 
alcanzaron un $34.8 \%$ en este rango. En términos más amplios se tiene que un $86.1 \%$ de las plantas con MAYORFLEX tuvo un costo de mano de obra menor al $41 \%$ y las plantas con MENORFLEX un $70.9 \%$.

Lo anterior indica que ahí donde es mayor la proliferación de formas de organización flexible la mano de obra representa un menor costo dentro del valor total de la producción. Por tanto, la mayor intensidad de capital en las plantas no sólo se debe al mayor uso de maquinaria, sino también de métodos de organización flexible, ya que éstos resultaron ser más "ahorradores de costos de mano de obra", incluso que ahí donde se incorpora tecnología dura.

CUADRO 5. Costo de la mano de obra en las plantas según su flexibilidad.

\begin{tabular}{lcccc}
\hline $\begin{array}{l}\text { Costo de la mano } \\
\text { de obra }\end{array}$ & \multicolumn{2}{c}{$\begin{array}{c}\text { Plantas con menor } \\
\text { flexibilidad }\end{array}$} & \multicolumn{2}{c}{$\begin{array}{c}\text { Plantas con mayor } \\
\text { flexibilidad }\end{array}$} \\
& No. & $\%$ & No. & $\%$ \\
\hline & 59 & 34.8 & 44 & 57.0 \\
Menos del 20\% & 62 & 36.1 & 23 & 29.1 \\
$21-40 \%$ & 50 & 29.2 & 11 & 13.9 \\
$41 \%$ y más & 171 & 100.0 & 78 & 100.0 \\
Total & & & & \\
\hline
\end{tabular}

FUENTE: Misma del cuadro 1.

\section{Estructura organizativa}

\section{a) Niveles jerárquicos}

El número de niveles o categorías dentro de la estructura escalafonaria no reportó grandes diferencias. El promedio para ambos tipos de plantas fue muy similar: 8.6 para las plantas con MENORFLEX y 9 para las plantas con MAYORFLEX. Las diferencias que sobresalen se refieren a la distribución de los niveles por rangos: hay proporcionalmente más plantas con estructuras organizacionales más simples en el caso de las de menor flexibilidad, que las de mayor flexibilidad. La mayoría de las plantas maquiladoras tienen menos de 11 niveles: $65.4 \%$ las plantas de MENORFLEX y $65.9 \%$ de las plantas con MAYORFLEX.

Sorprenden tres cuestiones: primera, las nulas diferencias encontradas en vista de que los métodos de organización flexible privilegian la simplificación 
de la estructura escalafonaria; segunda, que sea más simplificada la organización en plantas con 1 a 5 niveles, en las de menor flexibilidad; y tercero, que un importante número de plantas $-24.6 \%$ en las MENORFLEX y $34.1 \%$ en las de MAYORFLEX - tengan estructuras organizacionales "complejas" superiores a los 11 niveles.

CUADRO 6. Niveles jerárquicos en las plantas según su flexibilidad.

\begin{tabular}{|c|c|c|c|c|}
\hline \multirow{2}{*}{$\begin{array}{l}\text { Número de } \\
\text { niveles }\end{array}$} & \multicolumn{2}{|c|}{$\begin{array}{l}\text { Plantas con menor } \\
\text { flexibilidad }\end{array}$} & \multicolumn{2}{|c|}{$\begin{array}{l}\text { Plantas con mayor } \\
\text { flexibilidad }\end{array}$} \\
\hline & No. & $\%$ & No. & $\%$ \\
\hline $1-5$ & 77 & 37.7 & 10 & 13.2 \\
\hline $6-10$ & 77 & 37.7 & 41 & 52.7 \\
\hline Más de 10 & 51 & 24.6 & 27 & 34.1 \\
\hline Total & 205 & 100.0 & 78 & 100.0 \\
\hline Promedio & \multicolumn{2}{|c|}{8.58} & \multicolumn{2}{|c|}{9.0} \\
\hline
\end{tabular}

FUENTE: Misma del cuadro 1.

\section{b) Personal directo e indirecto}

La proporción del personal directo dentro del total del personal empleado de las plantas, tampoco reportó grandes diferencias. El promedio en ambos tipos de plantas fue muy similar: $76.2 \%$ en las plantas con MENORFLEX y $75.2 \%$ en las plantas con MAYORFLEX.

$\mathrm{Al}$ agrupar en rangos la proporción del personal directo se encontraron algunas diferencias. Aunque la mayoría de las plantas de uno y otro grado de flexibilidad se concentran en el rango intermedio, de 61 a $80 \%$, el porcentaje de plantas con MAYORFLEX en este rango fue mucho mayor (75.2\%) que el de las plantas con MENORFLEX (47.6\%). También se observan diferencias en otros rangos, lo que permite señalar que en las plantas con menor flexibilidad se encuentran los dos extremos: las que contratan relativamente más personal indirecto y las que prácticamente emplean sólo personal directo.

\section{c) Calificación}

La evaluación sobre la calificación respecto de puestos de trabajo seleccionados (operarios, supervisores y gerentes), está medida por la percepción de los propios gerentes entrevistados según la aplicación de una escala. 
CUADRO 7. Proporción de personal directo en las plantas según su flexibilidad.

\begin{tabular}{|c|c|c|c|c|}
\hline \multirow{2}{*}{$\begin{array}{l}\text { Proporción del per- } \\
\text { sonal directo sobre } \\
\text { el total empleado }\end{array}$} & \multicolumn{2}{|c|}{$\begin{array}{l}\text { Plantas con menor } \\
\text { flexibilidad }\end{array}$} & \multicolumn{2}{|c|}{$\begin{array}{l}\text { Plantas con mayor } \\
\text { flexibilidad }\end{array}$} \\
\hline & No. & $\%$ & No. & $\%$ \\
\hline $1-60 \%$ & 27 & 12.6 & 6 & 7.9 \\
\hline $61-80 \%$ & 100 & 47.6 & 59 & 75.2 \\
\hline $81 \%$ y más & 83 & 39.7 & 13 & 16.9 \\
\hline Total & 210 & 100.0 & 78 & 100.0 \\
\hline Promedio & \multicolumn{2}{|c|}{76.2} & \multicolumn{2}{|c|}{75.2} \\
\hline
\end{tabular}

FUENTE: Misma del cuadro 1.

En la calificación de los trabajadores operarios se observa una primera diferencia: en las plantas con MENORFLEX el porcentaje de trabajadores con este puesto que fueron considerados como calificados (24.6\%), es menor al porcentaje que se observa en las plantas con MAYORFLEX (37\%). En estas últimas, los operarios calificados resultaron ser el grupo más numeroso, en contraste con lo que sucede en las plantas con MENORFLEX donde el grupo más importante son los trabajadores escasamente calificados.

En el nivel representado por los supervisores, la mayoría de las plantas consideraron que su personal en este nivel era calificado: $59.8 \%$ en las plantas con MENORFLEX y $64.2 \%$ en las plantas con MAYORFLEX. Otro grupo importante de supervisores fueron considerados por las plantas como altamente calificados: $33 \%$ en las plantas con MENORFLEX y $35.8 \%$ en las plantas con MAYORFLEX. Llama la atención que en las plantas con MAYORFLEX no existan supervisores no calificados o escasamente calificados, lo que no sucede en las plantas con MENORFLEX donde estos grupos alcanzan en conjunto el $7.2 \%$.

En la categoría más alta, la de gerentes, la mayoría de plantas consideró que su personal en esta categoría era altamente calificado: $74.8 \%$ en las plantas con MENORFLEX y $75.2 \%$ en las plantas con MAYORFLEX. Los gerentes calificados representaron también un porcentaje considerable: $21.8 \%$ en las plantas con MENORFLEX y $24.8 \%$ en las plantas con MAYORFLEX. Sólo un grupo reducido de gerentes fue considerado como escasamente calificado y sólo para las plantas de MENORFLEX (3.4\%). 
CUADRO 8. Calificación de operarios, supervisores y gerentes de las plantas según su flexibilidad.

\begin{tabular}{|c|c|c|c|c|}
\hline & \multicolumn{2}{|c|}{$\begin{array}{l}\text { Plantas con menor } \\
\text { flexibilidad }\end{array}$} & \multicolumn{2}{|c|}{$\begin{array}{c}\text { Plantas con mayor } \\
\text { flexibilidad }\end{array}$} \\
\hline & No. & $\%$ & No. & $\%$ \\
\hline \multicolumn{5}{|l|}{ Gerentes } \\
\hline No calificado & 0 & 0.0 & 0 & 0.0 \\
\hline Escasamente calificado & 10 & 3.4 & 0 & 0.0 \\
\hline Calificados & 65 & 21.8 & 29 & 24.8 \\
\hline Altamente calificados & 223 & 74.8 & 88 & 75.2 \\
\hline Total & 298 & 100.0 & 117 & 100.0 \\
\hline \multicolumn{5}{|l|}{ Supervisores } \\
\hline No calificado & 1 & 0.4 & 0 & 0.0 \\
\hline Escasamente calificado & 18 & 6.8 & 0 & 0.0 \\
\hline Calificados & 158 & 59.8 & 79 & 64.2 \\
\hline Altamente calificados & 87 & 33.0 & 44 & 35.8 \\
\hline Total & 264 & 100.0 & 123 & 100.0 \\
\hline \multicolumn{5}{|l|}{ Operarios } \\
\hline No calificado & 91 & 28.5 & 39 & 30.7 \\
\hline Escasamente calificado & 147 & 46.1 & 41 & 32.3 \\
\hline Calificados & 78 & 24.5 & 47 & 37.0 \\
\hline Altamente calificados & 3 & 0.9 & 0 & 0.0 \\
\hline Total & 319 & 100.0 & 127 & 100.0 \\
\hline
\end{tabular}

FUENTE: Misma del cuadro 1.

En resumen, los resultados indican que, sin excepción, el personal de las plantas con MAYORFLEX tiene - según los gerentes - una calificación más alta, lo cual es consistente con la hipótesis que supone que es mayor la calificación en las plantas con organizaciones más eficientes o flexibles.

\section{d) Salarios}

Por último, en cuanto a los salarios semanales sin incluir prestaciones, los resultados fueron los siguientes:

En el nivel más bajo se observan que las plantas con MAYORFLEX pagan salarios ligeramente más altos que las plantas con MENORFLEX. El 75.4\% de las plantas con MENORFLEX paga más de 100,000 pesos semanales (en febrero de 1990) contra un $81.8 \%$ de las plantas con MAYORFLEX.

En el nivel representado por los supervisores, en términos generales, los salariois más altos estaban en las plantas con MENORFLEX. Un $29.2 \%$ 
de las plantas con MENORFLEX pagaban más de 500,000 pesos por semana, contra un $24.6 \%$ de las plantas con MAYORFLEX que pagan esa cantidad.

Finalmente, en el nivel más alto, representado por el puesto de gerentes, los salarios más altos se encuentran, en términos generales, en las plantas con MENORFLEX. Un $39.9 \%$ de estas plantas pagan un salario semanal de más de 500,000 pesos, frente al $24.4 \%$ de las plantas con MENORFLEX.

De acuerdo a estos datos tenemos que, con excepción de los operadores, las plantas con MENORFLEX pagan salarios más altos. Considerando la menor proporción de los costos de mano de obra en el costo total de la producción, estos resultados estarían indicando un uso más eficiente de la mano de obra en las plantas con MAYORFLEX.

CUADRO 9. Salarios según niveles.

\begin{tabular}{lrrrr}
\hline Salarios & \multicolumn{3}{c}{$\begin{array}{c}\text { Plantas con menor } \\
\text { flexibilidad }\end{array}$} & \multicolumn{2}{c}{$\begin{array}{c}\text { Plantas con mayor } \\
\text { flexibilidad }\end{array}$} \\
& \multicolumn{1}{c}{ No. } & $\%$ & No. & $\%$ \\
\hline & & & & \\
& & & & \\
Nivel más alto & 58 & 39.2 & 12 & 29.3 \\
$\quad$ De 100,000 a 500,000 & 31 & 20.9 & 19 & 46.3 \\
De 500,001 a 1,000,000 & 58 & 39.9 & 10 & 24.4 \\
Más de 1,000,000 & 148 & 100.0 & 41 & 100.0 \\
$\quad$ Total & & & & \\
Nivel intermedio & 104 & 70.8 & 46 & 75.4 \\
$\quad$ De 100,001 a 500,000 & 43 & 29.2 & 16 & 24.6 \\
Mas de 500,000 & 147 & 100.0 & 61 & 100.0 \\
$\quad$ total & & & & \\
Nivel más bajo & 56 & 24.6 & 14 & 18.2 \\
$\quad$ De 50,000 a 100,000 & 172 & 75.4 & 63 & 81.8 \\
Mas de 100,000 & 228 & 100.0 & 77 & 100.0 \\
Total & & & & \\
& & & & \\
\hline
\end{tabular}

FUENTE: Misma del cuadro 1.

\section{CONCLUSIONES}

La industria maquiladora de exportación en México no sólo es importante por los volúmenes de divisas y empleo que genera a nivel nacional, sino sobre todo por los impactos regionales. La IME sobresale dentro de la industria de la manufactura en México, por el dinamismo que ha mantenido, sobre todo a partir de 1982. Las características que definen actualmente 
a dicha industria son su dinamismo, la heterogeneidad y la reestructuración que vive. Entre los rasgos centrales de la reestructuración se encuentran la difusión de procesos automatizados, los cambios en el empleo y la proliferación de la flexibilidad organizacional, siendo algunas de las técnicas de organización que se implementan el trabajoen grupo, la rotación entre tareas, la multicalificación y los grupos de calidad, entre otros.

La heterogeneidad de la IME se observa en la existencia de tipos de maquiladoras que van, según su actividad, desde el ensamble hasta la manufactura; y según su flexibilidad, desde los sistemas de producción tayloristas hasta los "posfordistas". Un rasgo que sobresale en los diferentes tipos de maquila, y que es común a cllos, es el nivel de competitividad internacional al que están sujetos. En la IME se trabaja con estándares internacionales de calidad, lo que ha derivado en una amplia difusión del justo a tiempo y de unidades programables, fundamentalmente, máquinas-herramienta de control numérico. Un indicador que confirma estos estándares internacionales es el número de rechazos a la producción, en donde se encontró que la mayoría de las maquilas trabajan con un bajo indice de rechazos.

Muy probablemente, un fenómeno de mayor importancia que la automatización es la difusión de técnicas flexibles de organización del trabajo (denominadas también como tecnologías blandas). La IME se encuentra en un amplio proceso de reestructuración organizacional, sobre todo en lo que respecta a equipos de trabajo e involucramiento en el empleo; ambas técnicas alcanzaron a más del $50 \%$ de la mano de obra directa que participa en las mismas.

Finalmente, acerca de las diferencias entre plantas con mayor o menos flexibilidad se encontró que, referente a algunas características de las plantas, las de mayor flexibilidad son las de mayor tamaño en el empleo y son en las que el control de la mano de obra representa una menor proporción dentro del valor total de la producción. No se encontraron diferencias significativas en la antigüedad de las mismas.

Respecto a características sociodemográficas de la mano de obra, se puede concluir que en la mayoria de ellas no existen diferencias importantes como son la edad, los años de escolaridad, el origen rural o urbano y la experiencia laboral anterior. Sin embargo, se encontraron diferencias en la antigüedad y el sexo, resultando perfiles de gente más joven y porcentajes más clevados de hombres en las plantas con mayor flexibilidad organizacional.

Por último, acerca de la estructura organizativa se tiene que, en general, la flexibilidad y la estructura jerárquica, medida en número de niveles, no presenta grandes diferencias, al igual que la relación número de personal directo $\mathrm{c}$ indirecto. En donde sí se observan diferencias es en la percepción de la calificación por parte de los gerentes, ya que se puede establecer la 
proposición de que a mayor flexibilidad organizativa mayor calificación y, paradójicamente, a mayor salario menor flexibilidad organizacional.

Estos resultados permiten finalizar este documento mencionando que existe un proceso generalizado de flexibilidad organizacional en la IME, pero que éste no es homogéneo entre las distintas actividades económicas; al mismo tiempo se puede señalar que si bien hay diversas asociaciones entre flexibilidad y características de las plantas y de su estructura, casi no las hay en los perfiles socioeconómicos de la población empleada. Por lo que establecer determinaciones tecnológicas, en el perfil de la mano de obra por ejemplo, resulta incorrecto.

\section{BIBLIOGRAFÍA}

BROWN, F. y L. Domínguez. 1989. "Nuevas tecnologías en la industria maquiladora de exportación”. Comercio Exterior, no. 3, vol. 39, marzo.

CARRILLO V., Jorge. 1986. "Transformaciones en la industria maquiladora de exportación: ¿Una nueva fase? Cuadernos semestrales, Estados Unidos, no. 20, 2do. semestre de 1986.

- 1990. "Maquilización de la industria automotriz en México. De la industria terminal a la industria de ensamble", en: Jorge Carrillo V. (coordinador) La nueva era de la industria automotriz en México, Tijuana, El Colegio de la Frontera Norte.

GONZÁLEZ-ARÉCHIGA, Bernardo y Rocío Barajas Escamilla. 1987. "Las maquiladoras: ajuste estructural y desarrollo regional". Documentos de trabajo. OEKO-México, Resumen de un seminario internacional. SEP.

GONZÁLEZ-ARÉCHIGA, Bernardo y J. C. Ramírez. 1989. "Productividad sin distribución: cambio tecnológico en la maquiladora mexicana (1980-1986)", Frontera Norte, vol. 1, no. 1, enero-junio.

- 1990. Subcontratación y empresas transnacionales, COLEF-Fundación Ebert, Tijuana.

PALOMARES y Mertens. 1985. "El surgimiento de un nuevo tipo de trabajador en la industria de alta tecnología: el caso de la electrónica", en: Esthela Gutiérrez (coordinadora), Reestructuración productiva y clase obrera en México, Siglo XXI-UNAM (Testimonios de la crisis).

WILSON, Patricia. 1989. "The New Maquiladoras: Flexible Production in Low Wage Regions", University of Texas at Austin, Community and Regional Planning, Working Paper Series, no. 9, abril. Austin. 